\title{
BMJ Open Can we validate a clinical score to predict the risk of severe infection in patients with systemic lupus erythematosus? A longitudinal retrospective study in a British Cohort
}

\author{
Beatriz Tejera Segura, ${ }^{1}$ Iñigo Rua-Figueroa, ${ }^{2,3}$ Jose Maria Pego-Reigosa, ${ }^{4,5}$ \\ Victor del Campo, ${ }^{6}$ Chris Wincup, ${ }^{1}$ David Isenberg, ${ }^{\circ}$ Anisur Rahman ${ }^{1}$
}

To cite: Tejera Segura B, RuaFigueroa I, Pego-Reigosa JM, et al. Can we validate a clinical score to predict the risk of severe infection in patients with systemic lupus erythematosus? A longitudinal retrospective study in a British Cohort. BMJ Open 2019;9:e028697. doi:10.1136/ bmjopen-2018-028697

- Prepublication history for this paper is available online. To view these files, please visit the journal online (http://dx.doi. org/10.1136/bmjopen-2018028697).

Received 19 December 2018 Revised 9 March 2019 Accepted 15 May 2019

Check for updates

(c) Author(s) (or their employer(s)) 2019. Re-use permitted under CC BY-NC. No commercial re-use. See rights and permissions. Published by BMJ.

For numbered affiliations see end of article.

Correspondence to

Dr Anisur Rahman;

anisur.rahman@ucl.ac.uk

\section{ABSTRACT}

Objective Severe infections are a major cause of morbidity and mortality in patients with systemic lupus erythematosus (SLE). Our primary objective was to use data from a large Spanish cohort to develop a risk score for severe infection in SLE, the SLE Severe Infection Score (SLESIS) and to validate SLESIS in a separate cohort of 699 British patients.

Design and setting Retrospective longitudinal study in a specialist tertiary care clinic in London, UK.

Participants Patients fulfilling international classification criteria for SLE $(n=209)$. This included 98 patients who had suffered severe infections (defined as infection leading to hospitalisation and/or death) and 111 randomly selected patients who had never suffered severe infections. Outcomes We retrospectively calculated SLESIS at diagnosis for all 209 patients. For the infection cases we also calculated SLESIS just prior to infection and compared it to SLESIS in 98 controls matched for disease duration. We carried out receiver operator characteristic (ROC) analysis to quantify predictive value of SLESIS for severe infection.

Results Median SLESIS (IQR) at diagnosis was higher in the infection group than in the control group (4.27 (3.18) vs 2.55 (3.79), $p=0.0008)$. Median SLESIS prior to infection was higher than at diagnosis (6.64 vs $4.27, p<0.001)$. In ROC analysis, predictive value of SLESIS just before the infection (area under the curve $(A U C)=0.79$ ) was higher than that of SLESIS at diagnosis (AUC=0.63).

Conclusions We validated the association of SLESIS with severe infection in an independent cohort. Calculation of SLESIS at each clinic visit may help in management of infection risk in patients with SLE. Prospective studies are needed to confirm these findings.

\section{INTRODUCTION}

Systemic lupus erythematosus (SLE) is a systemic autoimmune disease that mainly presents in women aged between 30 years and 50 years. Infection is a major cause of morbidity and mortality in patients with SLE, being responsible for $11 \%-45 \%$ of hospitalisations

\section{Strengths and limitations of this study}

- We developed an algorithm (Systemic Lupus Erythematosus Severe Infection Score (SLESIS)) designed to predict the risk of severe infections in patients with systemic lupus erythematosus (SLE) that could be a useful tool in clinical practice.

- SLESIS was developed using data from a Spanish lupus registry (Spanish Society of Rheumatology Lupus Registry (RELESSER)) that includes almost 4000 patients with SLE.

- We applied the algorithm in a British cohort of 699 patients with SLE in order to validate it.

- The algorithm consists of seven variables that are all obtainable from information obtained in routine clinical practice.

- We acknowledge several limitations: the retrospective design of the study, the different ethnicity in the Spanish and British populations and the Katz Index as an item of the score.

as well as $20 \%-55 \%$ of deaths, according to different studies. ${ }^{1-6}$

There are immunological and genetic disorders that predispose to the development of infections in patients with SLE. ${ }^{78}$ Respiratory tract infections are the most common infections in these patients, and bacteraemia the leading cause of mortality. Regarding the aetiology, pathogenic bacteria are most frequently involved in the infection, followed by viruses and fungi. ${ }^{9} 10$

There are several well-established risk factors for infection such as age at diagnosis, male sex, ethnicity and use of corticosteroids, among others; however, the independent contribution of each factor is not well understood. ${ }^{11-14}$ On the other hand, severe infections have been associated with decreased survival of patients with SLE, acting as an 
independent risk factor for mortality at 10 years after diagnosis. $^{2-4} 14$

This information about the risk factors associated with infection in SLE has not yet been combined into tools to estimate the risk of severe infection in individual patients over time. The development of an algorithm for predicting the risk of severe infection could be very useful to monitor risk factors more closely in a weighted way. It could allow us to implement prophylactic measures such as vaccination in the highest-risk patients and to consider early intervention such as antibiotic therapy in this group of patients with the ultimate goal of reducing morbidity and improving survival. ${ }^{15-17}$ Similar scores to predict risk of severe infection have been developed and validated in patients with rheumatoid arthritis (RA) with encouraging results. ${ }^{18}$

In a recent original study from the Spanish Society of Rheumatology Lupus Registry (RELESSER), the authors studied 3658 patients with SLE, of whom 705 (19.3\%) had suffered one or more severe infections. A multivariable Cox regression model for repeated events (Andersen-Gill) was used to define the impact of a range of demographic and clinical variables on the risk of developing severe infection, expressed as HR of developing severe infection for patients with each associated factor, compared with those without that factor. ${ }^{17}$ Seven factors had an HR more than 1.

In the study reported in the current paper these seven factors were used to generate a score-the Systemic Lupus Erythematosus Severe Infection Score (SLESIS), designed to predict the risk that a patient with lupus will develop a severe infection.

The overarching primary objective of the current study was to validate SLESIS in an independent population of patients with SLE from London, UK.

In the validation cohort, we aimed to answer three questions.

1. Does SLESIS at the time of diagnosis predict future severe infection?

2. In patients who suffer a severe infection, does SLESIS change between diagnosis and the time of infection?

3. Does SLESIS just prior to an infection predict future severe infection?
If (2) and (3) are true, it could support assessing SLESIS at every appointment rather than only at diagnosis.

\section{PATIENTS AND METHODS}

\section{Development and definition of SLESIS}

SLESIS was developed using variables related to infection from the RELESSER cohort of 3658 patients with SLE who meet $\geq 4$ critera of the American College of Rheumatology (ACR) classification criteria for SLE. The variables, definitions and methodological characteristics of the RELESSER registry have previously been described in detail. $^{19} 20$

Table 1 shows the seven factors that were found to be associated with increased risk of developing severe infection (defined as infection leading to hospitalisation and/or death) in the RELESSER paper ${ }^{17}$ together with HR for severe infection associated with each factor. There are four factors that are graded present or absent; age at diagnosis $>46$ years $(\mathrm{HR}=1.12)$; Latin American ethnicity $(\mathrm{HR}=2.4)$; dose of corticosteroid $\geq 10 \mathrm{mg}$ /day $(\mathrm{HR}=1.33)$; and male sex $(\mathrm{HR}=1.49)$. For each of these factors, the contribution to SLESIS is either 0 (if absent) or $1 \times \mathrm{HR}$ (if present).

One factor (Katz Index ${ }^{21}$ of disease severity) is a continuous variable. The contribution of this factor is its absolute value $\times 1.06$ (its $\mathrm{HR}$ ).

The other factors are previous hospitalisations for SLE $(\mathrm{HR}=2.73)$ and previous severe infection $(\mathrm{HR}=2.40)$. For these factors the contribution to SLESIS is the number of events $\times$ HR.

Thus SLESIS for a male Latin American patient, diagnosed at age 22 years, taking $12 \mathrm{mg}$ prednisolone per day with a Katz Index of 2, two previous hospitalisations for SLE and no previous severe infections would be calculated as follows.

$(0 \times 1.12)+(1 \times 2.4)+(1 \times 1.33)+(1 \times 1.49)+(2 \times 1.06)$ $+(2 \times 2.73)+(0 \times 2.40)=11.31$.

Conversely SLESIS for a female Chinese patient, diagnosed at age 50 years, taking $5 \mathrm{mg}$ prednisolone per day with a Katz Index of 2, no previous hospitalisations for SLE and three previous severe infections would be calculated as follows.

\begin{tabular}{|c|c|c|c|}
\hline Variable & $\beta$ coefficient & HR for severe infection & $95 \% \mathrm{Cl}$ \\
\hline Age at diagnosis ( $>46$ years) & 0.1163 & 1.12 & 1.07 to 1.18 \\
\hline Latin American ethnicity & 0.427 & 2.40 & 2.29 to 2.5 \\
\hline Current dose of corticosteroids $\geq 10 \mathrm{mg} /$ day & 0.2878 & 1.33 & 1.15 to 1.55 \\
\hline Sex (male) & 0.3692 & 1.49 & 1.22 to 1.81 \\
\hline Previous hospitalisation for SLE & 1.0049 & 2.73 & 2.22 to 3.35 \\
\hline Katz Index ${ }^{21}$ & 0.062 & 1.06 & 1.03 to 1.1 \\
\hline Each previous severe infection & 0.8739 & 2.40 & 2.29 to 2.50 \\
\hline
\end{tabular}

These HR values were derived from the retrospective analysis of 3658 Spanish patients described in ref [17].

SLE, systemic lupus erythematosus; SLESIS, Systemic Lupus Erythematosus Severe Infection Score. 
$(1 \times 1.12)+(0 \times 2.4)+(0 \times 1.33)+(0 \times 1.49)+(2 \times 1.06)$ $+(0 \times 2.73)+(3 \times 2.40)=10.44$.

\section{Validation cohort}

Validation was carried out in the lupus clinic at University College London Hospital (UCLH). All patients fulfilled the 1997 revised criteria of ACR for the classification of SLE. ${ }^{22}$ The clinic has been running continuously since 1979 and has records from 699 patients. Medical records of all the patients were reviewed to identify those who had suffered severe infections. Severe infection was defined as that leading to hospitalisation and/or death. Isolation of the causative agent was not required in every case, with final classification as an infection being made using standard clinical criteria as in the previous RELESSER Study. ${ }^{17}$ Only infections recorded during the follow-up period (ie, after the diagnosis of lupus) were included as outcomes.

We used a nested case-control design. We identified 98 patients who had suffered at least one severe infection and compared their medical records with those of 111 randomly selected patients with SLE who had never suffered from severe infections. By retrospective analysis of medical records, we obtained comprehensive information about demographic, clinical, serological and treatment factors in these two groups.

We calculated SLESIS for each of the 209 patients at the time of diagnosis. For each of the 98 patients with infection we also calculated SLESIS for the clinical assessment carried out at the last consultation prior to the infection. For comparison, we calculated SLESIS in 98 controls matched for disease duration, as follows. For each infection case we selected one control case from the 111 who had never suffered severe infections. For the control we calculated SLESIS at the time point after diagnosis corresponding to the duration after diagnosis when the matched case suffered severe infection.

\section{Statistical analysis}

Demographic and clinical characteristics were compared between patients who developed severe infection and patients who did not, using a Pearson $\chi^{2}$ for categorical variables or a Student's t-test for normally distributed continuous variables (data expressed as mean $\pm \mathrm{SD}$ ). For non-normally distributed variables, either a MannWhitney $\mathrm{U}$ test or a logarithmic transformation was performed, and data are expressed as median and IQR. Multivariable logistic regression analysis was performed to establish which factors were independently associated with severe infection.

We compared SLESIS scores between case and control groups using the Mann-Whitney $\mathrm{U}$ test. Receiver operator characteristics (ROC) analysis was used to define the area under the curve (AUC) for SLESIS at diagnosis and SLESIS just prior to infection. We also identified several possible cut-off scores for SLESIS with different combinations of specificity and sensitivity for severe infection.
The STATA for Windows statistical software package (V.13.1) was used for all statistical analysis. Significance was defined as $\mathrm{p}<0.05$.

\section{Patients and/or public involvement}

Patients and/or public were not involved in this study.

\section{RESULTS}

Table 2 shows a comparison of 98 patients (14\% of the total cohort of 699 patients) who developed severe infection and a control group of 111 patients who never suffered from severe infection. These two groups did not differ in age, sex or ethnicity.

Patients who developed severe infections after diagnosis of SLE were more likely to have suffered previous severe infections before diagnosis of SLE (Pearson $\chi^{2}=11.69$, $\mathrm{p}=0.001$ ) or to have been hospitalised for SLE (Pearson $\left.\chi^{2}=11.89, p=0.001\right)$ compared with those patients who never had severe infections. In terms of SLE manifestations, skin and renal disease were more common in the infection group (Pearson $\chi^{2}=6.25, \mathrm{p}=0.012$; Pearson $\left.\chi^{2}=9.5, \mathrm{p}=0.004\right)$ whereas joint involvement was more frequent in the non-infection group (Pearson $\chi^{2}=11.21$, $\mathrm{p}=0.001$ ).

Patients in the infection group were more likely to have been treated with high doses of corticosteroids or an immunosuppressant (IS). Whereas 55\% of the infection group had ever been treated with corticosteroids at a dose $\geq 10 \mathrm{mg}$ per day, this only happened in $22 \%$ of the non-infected group (Pearson $\chi^{2}=10.54, p=0.009$ ). Significantly increased risk of infection was found for azathioprine (Pearson $\chi^{2}=5.89 \mathrm{p}=0.015$ ) and cyclophosphamide (Pearson $\chi^{2}=8.26, p=0.004$ ) but not for mycophenolate or rituximab. Hydroxychloroquine (HCQ) had been taken by $77 \%$ of the non-infection group compared with $67 \%$ of the infection group with no significant difference between groups.

Regarding laboratory data, only neutropenia, which was twice as common in the infection group ( $34 \%$ vs $17 \%$ ), was significantly different between both groups (Pearson $\left.\chi^{2}=7.63, \mathrm{p}=0.006\right)$.

After multivariable analysis adjusted for age, only previous infection ( $\beta$ coefficient: $2.20,95 \%$ CI 0.77 to 3.62 ; $\mathrm{p}=0.003$ ), previous hospitalisations for SLE ( $\beta$ coefficient: $1.12,95 \%$ CI 0.19 to $2.07 ; \mathrm{p}=0.021)$ and treatment with corticosteroids $\geq 10 \mathrm{mg} /$ day, ( $\beta$ coefficient: $3.39,95 \%$ CI 2.33 to $4.44 ; \mathrm{p}<0.001)$ remained statistically significant.

\section{Type of infection}

The most common type of severe infection was respiratory tract infection $(\mathrm{n}=29 ; 29.6 \%$ of all infections), followed by urinary tract infection $(\mathrm{n}=22,22.4 \%)$ and gastrointestinal infection $(n=22,22.4 \%)$. Less frequent were soft tissue infection $(\mathrm{n}=11,11.2 \%)$ and nervous system infections $(n=4,4.1 \%)$. Twelve $(12.4 \%)$ of the patients developed documented bacteraemia on blood culture and 15 $(15.3 \%)$ had multiple organ involvement. 
Table 2 Descriptive data: patients with systemic lupus erythematosus (SLE) with infection versus without infection

Study (infection) group Control (non-infection) $(\mathrm{n}=98)$ group $(n=111)$

P values

\begin{tabular}{|c|c|c|c|}
\hline Gender, n (\%) & $90(92)$ & $103(93)$ & \\
\hline Female (male) & $8(8)$ & $8(7)$ & ns \\
\hline Median age at diagnosis of SLE (IQR), years & $30.5(27)$ & $31(18)$ & ns \\
\hline Median age at time of infection (IQR), years & $43(25)$ & Not applicable & \\
\hline \multicolumn{4}{|l|}{ Ethnicity, n (\%) } \\
\hline Caucasian & $48(49)$ & $72(65)$ & ns \\
\hline Hispanics & $3(3)$ & $2(2)$ & \\
\hline Afro-Caribbean & $28(29)$ & $20(18)$ & \\
\hline Asian & $7(7)$ & $6(5)$ & \\
\hline Other & $12(12)$ & $12(11)$ & \\
\hline Median length of follow-up (IQR), years & $9.5(14)$ & $14(9)$ & ns \\
\hline Previous infection before SLE diagnosis,n (\%) & $16(16)$ & $3(2)$ & 0.001 \\
\hline Previous hospitalisations due to SLE n (\%) & $55(56)$ & $36(32)$ & 0.001 \\
\hline \multicolumn{4}{|l|}{ SLE main features (cumulative) $n,(\%)$} \\
\hline Skin disease & $76(78)$ & $67(60)$ & 0.012 \\
\hline Joint disease & $72(74)$ & $101(91)$ & 0.001 \\
\hline Renal disease & $42(43)$ & $24(22)$ & 0.004 \\
\hline Neuropsychiatric disease & $13(13)$ & $12(11)$ & ns \\
\hline Serositis & $19(19)$ & $33(30)$ & ns \\
\hline \multicolumn{4}{|l|}{ Previous drug treatment $\mathrm{n}(\%)$} \\
\hline Corticosteroids & $89(91)$ & $43(39)$ & $<0.001$ \\
\hline Corticosteroids ( $\geq 10 \mathrm{mg}$ per day at any time) & $54(55)$ & $25(23)$ & 0.009 \\
\hline No hydroxychloroquine & $32(33)$ & $25(23)$ & ns \\
\hline Azathioprine & $47(48)$ & $35(32)$ & 0.015 \\
\hline Mycophenolate & $36(35)$ & $28(25)$ & ns \\
\hline Cyclophosphamide & $28(29)$ & $13(13)$ & 0.004 \\
\hline Rituximab & $26(27)$ & $25(23)$ & ns \\
\hline \multicolumn{4}{|l|}{ Laboratory data, $\mathrm{n}(\%)$ at any time } \\
\hline Neutropenia & $33(34)$ & $19(17)$ & 0.006 \\
\hline Lymphopenia & $76(78)$ & $73(66)$ & ns \\
\hline Anti-dsDNA + & $67(68)$ & $66(60)$ & ns \\
\hline Low C3 & $57(58)$ & $50(45)$ & ns \\
\hline \multicolumn{4}{|l|}{ Outcomes } \\
\hline Deaths & $26(27)$ & $2(2)$ & \\
\hline Patients with $>1$ infection & $18(18)$ & 0 & 0.03 \\
\hline
\end{tabular}

\section{Mortality}

About a quarter (26.5\%) of the patients died during their admission for severe infection, though this does not imply that death was always due to infection as many patients had comorbidities or active lupus as well. Having recorded this high proportion of deaths, we analysed the differences between the group of 26 patients who died during their admission for infection and the 72 patients who survived. These results are summarised in table 3 .
Patients who did not die had more previous hospitalisations due to SLE, compared with those who died (Pearson $\chi^{2}=33.71, p=<0.001$ ). In contrast, those who died were more likely to have suffered from severe infections prior to the diagnosis of SLE (Pearson $\chi^{2}=8.66$, $\mathrm{p}=0.003$ ).

There were no statistically significant differences among the clinical manifestations of SLE between these two groups. 
Table 3 Comparison of patients who died during admission for severe infection and those who survived

\section{Deceased patients $\mathrm{n}=\mathbf{2 6}$ Alive patients $\mathrm{n}=\mathbf{7 2}$}

$(26.5 \%)$

(73.5\%)

P values

\begin{tabular}{|c|c|c|c|}
\hline Mean age at diagnosis (SD) & $33.8(14.1)$ & $31.2(15.1)$ & ns \\
\hline Mean age at infection (SD) & $42.88(16.7)$ & $40.88(16.7)$ & ns \\
\hline Follow-up between diagnosis and first infection (years) & $9.11(7.04)$ & $10.8(10.3)$ & ns \\
\hline \multicolumn{4}{|l|}{ Ethnicity n, (\%) } \\
\hline Caucasian & $16(61.5)$ & $31(43.1)$ & ns \\
\hline Hispanics & NA & $3(4.2)$ & \\
\hline Afro-Caribbean & $8(30.8)$ & $20(27.8)$ & \\
\hline Asian & $2(7.7)$ & $4(5.6)$ & \\
\hline Others & NA & $12(16.7)$ & \\
\hline Gender (female), n (\%) & $25(96)$ & $65(90)$ & ns \\
\hline Previous hospitalisations, $\mathrm{n}(\%)$ & $2(8)$ & $53(74)$ & $<0.001$ \\
\hline Previous severe infection before diagnosis of SLE, $n$ (\%) & $9(35)$ & $7(10)$ & 0.003 \\
\hline Skin disease, $\mathrm{n}(\%)$ & $18(69)$ & $58(81)$ & ns \\
\hline Renal disease, $\mathrm{n}(\%)$ & $10(39)$ & $32(44)$ & ns \\
\hline Joint disease, $n(\%)$ & $22(85)$ & $50(69)$ & ns \\
\hline Neuropsychiatric disease, $\mathrm{n}(\%)$ & $4(15)$ & $9(13)$ & ns \\
\hline Serositis, n (\%) & $7(27)$ & $12(17)$ & ns \\
\hline Corticosteroids per day at any time), n (\%) & $20(77)$ & $69(96)$ & 0.02 \\
\hline Corticosteroids $\geq 10 \mathrm{mg} /$ day at any time, $\mathrm{n}(\%)$ & $17(65)$ & $37(51)$ & ns \\
\hline Hydroxychloroquine, n (\%) & $20(77)$ & $46(64)$ & ns \\
\hline Rituximab, n (\%) & $1(4)$ & $25(35)$ & 0.002 \\
\hline Cyclophosphamide, n (\%) & $11(42)$ & $17(24)$ & ns \\
\hline Azathioprine, n (\%) & $14(54)$ & $33(46)$ & ns \\
\hline Mycophenolate, n (\%) & $10(39)$ & $26(36)$ & ns \\
\hline Splenectomy, n (\%) & 0 & $3(4)$ & ns \\
\hline Bacteraemia, n (\%) & $4(15)$ & $9(13)$ & ns \\
\hline Multiple organ involvement, $\mathrm{n}(\%)$ & $12(46)$ & $5(7)$ & $<0.001$ \\
\hline Low complement, n (\%) & $11(42)$ & $46(64)$ & ns \\
\hline Neutropenia, n (\%) & $5(19)$ & $28(39)$ & ns \\
\hline Lymphopenia, n (\%) & $20(77)$ & $56(78)$ & ns \\
\hline a-dsDNA, n (\%) & $20(77)$ & $47(65)$ & ns \\
\hline
\end{tabular}

a-dsDNA, Anti-double stranded DNAantibodies; SLE, systemic lupus erythematosus.

Regarding treatment, there were statistically significant differences for steroids at any time (Pearson $\chi^{2}=8.19$, $\mathrm{p}=0.004$ ) and rituximab (Pearson $\chi^{2}=9.34, \mathrm{p}=0.002$ ). However, for both these variables, patients who survived were more likely to have taken these drugs than those who died. Importantly, we only considered use of drugs in the period between diagnosis and first infection so survival from first infection would not affect the proportion of patients recorded as taking any drug.

Multiple organ involvement by infection was more common in deceased patients than in patients who survived (Pearson $\chi^{2}=20.5, p=<0.001$ ). Patients who died were more likely to have had positive anti-dsDNA compared with those who survived.
After multivariable analysis adjusted for age, previous hospitalisations due to SLE, previous infection and the presence of multiple organ involvement remained statistically significant as factors differentiating patients who died from those who survived ( $\beta$ coefficient: $-6.15,95 \%$ CI -9.57 to $-2.73 ; p<0.001 ; \beta$ coefficient: $3.55,95 \%$ CI 0.47 to 6.63; $\mathrm{p}=0.024$ and $\beta$ coefficient $3.5195 \%$ CI 0.9 to 6.12 ; $\mathrm{p}=0.008$ ).

\section{Analysis of SLESIS values over time}

Median (IQR) SLESIS at diagnosis of SLE in patients with infection was 4.27 (3.18) which was significantly higher than in the control (no infection) group (median 2.55, IQR $3.79(\mathrm{z}=-3.34 ; \mathrm{p}=0.0008)$. Median (IQR) SLESIS 


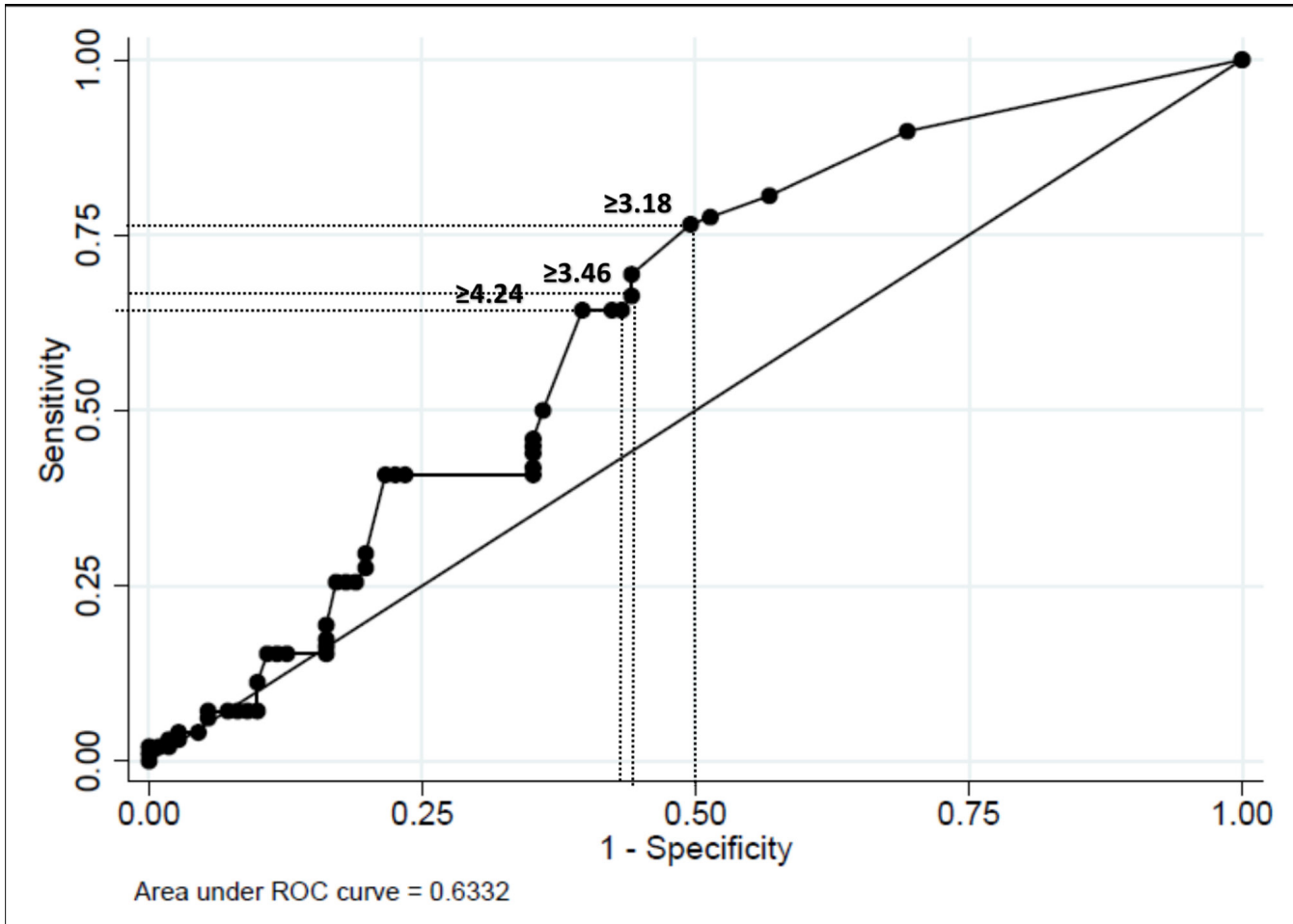

Figure 1 Receiver operator characteristics (ROC) curve analysis-Systemic Lupus Erythematosus Severe Infection Score (SLESIS) at diagnosis.

just before the infection in the infection group was 6.64 (4.18) which was statistically higher than the median SLESIS at diagnosis in those patients $(\mathrm{z}=-5.73, \mathrm{p}=<0.001)$ and also higher than median SLESIS in the duration-matched control group at a comparable time point in their disease course (median 3.32, IQR 3.16, $\mathrm{z}=-6.99$; $\mathrm{p}=<0.001)$. There were no statistically significant differences between the first and second SLESIS values (ie, diagnosis and comparable time point to infection) for the matched controls.

Figure 1 shows the ROC analysis for SLESIS at diagnosis. The AUC was 0.6332 (SD 0.04; 95\% CI 0.56 to 0.70 ). We defined three possible cut-offs to distinguish patients with and without severe infection. We selected the cut-offs which had classified correctly the maximum percentage of the sample taking into account sensitivity and specificity. A cut-off for SLESIS at diagnosis $\geq 3.18$ identified patients who would develop severe infection with sensitivity $77 \%$ and specificity $51 \%$. For SLESIS $\geq 3.46$, sensitivity was $66 \%$ and specificity $55 \%$. For SLESIS $\geq 4.24$, sensitivity was $64 \%$ and specificity $60 \%$.

Figure 2 shows the ROC curve for SLESIS just prior to the infection. The AUC was 0.79 (SD 0.03 95\% CI 0.73 to 0.85 ), which was better than for SLESIS at diagnosis. We selected three cut-offs: $\geq 3.67$ identified patients who would develop severe infection with sensitivity of $90 \%$ and specificity of $43 \%$. Similar results were obtained using $\geq 3.79$ as a cut-off (sensitivity of $88 \%$, specificity of $50 \%$ ). For SLESIS $\geq 4.24$, sensitivity was $86 \%$ and specificity $60 \%$.

\section{DISCUSSION}

In patients with SLE it has been reported that the prevalence of life-threatening infections appears to be highest within the first 5 years after disease onset. ${ }^{23}$ However, severe infection represents a threat throughout the course of the disease, especially when these patients become older and develop more comorbidities related to age. Given the potential mortality in patients with SLE due to such infections, it is pertinent to pursue new options that allow us to predict which groups of patients will have higher risk of developing major infections. Our aim was to develop a risk score (SLESIS) to aid in this purpose. This study fulfils the important task of testing the properties of this SLESIS score in a cohort independent of the RELESSER $^{19} 20$ population in which it was developed. Our London cohort differs from the Spanish cohort in terms of ethnicity but SLESIS still exhibits a valid and statistically significant association with severe infection.

During the last few years, there have been several publications aiming to develop algorithms that could measure the risk of developing severe infections in patients with other autoimmune diseases. Crowson $e t a l^{24}$ developed a score in order to detect severe infection promptly in a cohort of 410 patients with RA. The score was designed and validated to predict the 1-year risk of severe infection whereas SLESIS was elaborated to predict the risk of severe infection ever. Zink $e t a l^{18}$ built a score based on the Rheumatoid Arthritis Observation of Biologic Therapy cohort in order to predict serious infection in patients on biologic treatment, with promising results. However, 


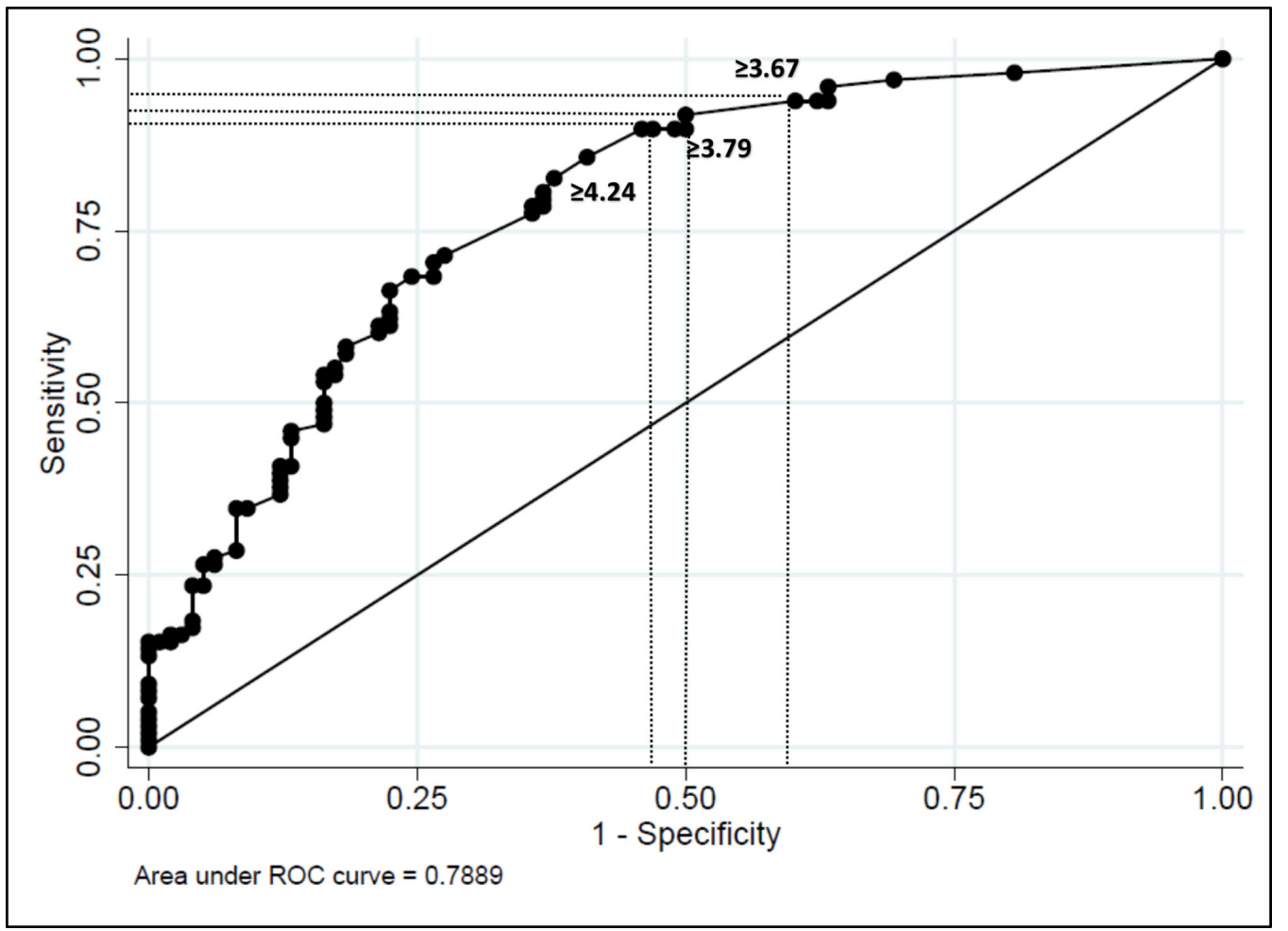

Figure 2 Receiver operator characteristics (ROC) curve analysis Systemic Lupus Erythematosus Severe Infection Score (SLESIS) before infection.

the authors suggested that their risk score was valid only in Caucasian populations in Western Europe. Beca et $a l^{25}$ designed a risk calculator algorithm to differentiate flares from infections in patients with SLE with fever. The study encompassed 130 patients with SLE who presented with fever over a period of 13 years. ROC curve analysis in both papers showed that both scores were able to predict infection with an AUC between 0.8 and 0.9. Nonetheless, both studies validated the algorithms in similar cohorts in terms of ethnicity distribution compared with the original cohorts. In contrast, we selected a validation cohort of patients in a different country, with a different range of ethnicities. In the Spanish cohort, $93 \%$ of patients were Caucasian and fewer than $1 \%$ African/Caribbean. In London, 68\% were Caucasian and 28\% African/ Caribbean, an ethnic group recognised as suffering more severe SLE.

Patients with previous severe infection were predisposed to developing major infection and were also more likely to die during admission for severe infection. Impaired immune system functions in a subgroup of patients with SLE may contribute to increase the infection risk in those patients. There are immunological and genetic disorders that predispose to the development of infections in patients with SLE. Furthermore, other clinical and treatment factors might play a crucial role in the predisposition of patients with SLE to develop infection. For instance, the infection group were more likely than the controls to have had previous hospitalisations for SLE. These findings are comparable to other previous reports. ${ }^{26-28}$ In contrast, in the study of Ruiz-Irastorza $e t$ al, lung disease was the only clinical manifestation that was considered a predictor of major infection after the multivariate analysis. ${ }^{29}$

In carrying out this project, we noted the fact that a quarter of the patients recorded as suffering severe infections died during their admission for infection. To investigate this mortality further, we carried out univariable and multivariable analyses of factors that could potentially influence whether or not these patients died. Surprisingly, though previous hospitalisation due to SLE was a factor independently associated with increased risk of severe infection it was also associated with increased chance of surviving that severe infection, even after multivariable analysis. There is no obvious explanation for this apparent paradox. Perhaps the threshold for starting antibiotics was lower in such patients. We considered the possibility that deaths from severe infection had all occurred many years ago, during a period when more patients were treated with high-dose IS such as cyclophosphamide. However, this is not the case as 12 patients died between 2000 and 2012 while the other 14 died before the year 2000 .

SLESIS was significantly higher just before the severe infection in comparison to the value at the diagnosis of SLE. This reinforces the idea that repeated measurement of SLESIS may be a helpful clinical assessment tool. As lupus activity and treatment change over time, it is predictable that SLESIS would change over time, but it was important to investigate whether this makes SLESIS at diagnosis a poorer predictor of infection. Development of chronic damage over time, as well as cumulative 
use of corticosteroids and immunosuppressive drugs, could contribute to these changes in SLESIS related to increased infection risk. Some variables of SLESIS will never change over time, such as age at diagnosis, ethnicity and gender, whereas others, such as number of previous severe infections, Katz Index and current dose of corticosteroids, could change and may thus contribute to changes in SLESIS. One strategy could be to recalculate SLESIS annually and after every severe infection and hospitalisation. In this way, a prospective study could be carried out to calculate SLESIS multiple times during follow-up in order to assess its variation over time and see if its peak (vs lower values during follow-up) is really associated with a higher probability of severe infection.

In line with other reports, ${ }^{3031}$ we found that ever-use of corticosteroids $\geq 10 \mathrm{mg}$ /day was associated with increased infection risk. In fact, more than $50 \%$ of patients of our cohort who suffered from severe infections had been treated with $\geq 10 \mathrm{mg}$ /day of corticosteroids. Other immunosuppressive treatments have been associated with high risk of infections although results have been controversial among the different cohorts. ${ }^{31}$ In our study, cyclophosphamide and azathioprine were associated with increased risk of severe infection in the univariate analysis but that significance was lost after the multivariable analysis.

There was a trend with HCQ which seemed to be the opposite to corticosteroids or IS, since fewer patients in the study group than the control group had ever been treated with HCQ. This finding is in agreement with previous reports ${ }^{29} 32$ although in our study, it did not reach statistical significance. Ruiz-Irastorza $e t a t^{29}$ found that patients taking antimalarials were 16 times less likely to suffer a major infection. Similarly, Sisó $e t a l^{32}$, found a lower frequency of infections among those previously treated with antimalarials in a retrospective study. Bultink et al, in a study designed to analyse the effect of the deficiency of functional mannose-binding lectin, also found that treatment with HCQ was associated with fewer severe infections although the authors attributed this association to the presumed lesser severity of patients with lupus who take HCQ. ${ }^{33}$

Overall, the AUCs in our study were not as high as for previous scores developed for RA. ${ }^{18}{ }^{24}$ However, this is the first score that has been developed to predict severe infections in SLE and may represent the first step in developing tools to do this accurately. We acknowledge several limitations in our study. First is the retrospective design of the study. Second, the ethnicity item in SLESIS distinguishes only Latin-American ethnicity as a separate group, which was not helpful in the London population. A modified SLESIS including HR for other ethnic groups would be more widely applicable globally but could not have been derived from the RELESSER cohort (too ethnically homogeneous) or the UCLH cohort (too small). Thirdly, the Katz Index used by RELESSER is a simple and helpful measure of disease severity (not just activity) but is not widely used in clinical practice. However, in the original RELESSER Study, ${ }^{17}$ from which SLESIS was derived, Systemic Lupus International Collaborating Clinics (SLICC)/ACR/Damage Index, Katz Index and SELENA-SLEDAI (Safety of Estrogen in Lupus National Assessmentstudy-Systemic Lupus Erythematosus Disease Activity Index) activity score were all measured and the Katz Index was the only one statistically associated with severe infection. Notwithstanding these limitations, we have shown that SLESIS at diagnosis was significantly higher in the group of patients who later suffered severe infections than in the control group of patients with SLE who did not suffer severe infection.

How might SLESIS be used in clinical practice? It is important to bear in mind that both sensitivity and specificity for severe infection were modest for SLESIS at diagnosis. For SLESIS just before the infection, sensitivity was high $(90 \%)$ but specificity was low $(54 \%)$. The low specificity means that SLESIS could not be used to decide on use of management measures with a high risk of adverse effects. We would suggest that patients with high SLESIS $(>3.5)$ at any point could be followed up with a higher index of suspicion for infection and lower threshold for using antibiotics. Although this may be an obvious extrapolation of the data, it is not possible to conclude that the early use of antibiotics in patients with high SLESIS will impact on the outcomes, and therefore, this strategy cannot be suggested based solely on the results of our work. SLESIS at diagnosis, together with other factors, might also help in deciding which patients might benefit from vaccination against bacterial infections (eg, pneumococcus) and considering a benefit-risk balance of corticosteroids ( $\geq 10 \mathrm{mg} /$ day) and other IS.

In summary, despite the limitations, our study has demonstrated strong arguments to perform future prospective studies to assess if SLESIS, or an improved form of SLESIS, could be clinically useful in the early diagnosis and prevention of severe infections in patients with SLE.

\section{Author affiliations}

${ }^{1}$ Centre for Rheumatology Research, University College London, London, UK ${ }^{2}$ Rheumatology Department, Doctor Negrín University Hospital of Gran Canaria, Las Palmas de Gran Canaria, Spain

${ }^{3}$ RELESSER Study Group, Spain

${ }^{4}$ Rheumatology Department, University Hospital Vigo, IRIDIS Group, Instituto de Investigación Sanitaria Galicia Sur (IISGS), Vigo, Spain

${ }^{5}$ RELESSER Study Group, Spain

${ }^{6}$ Preventive Medicine and Epidemiology Department, University Hospital Vigo, IRIDIS Group, Instituto de Investigación Sanitaria Galicia Sur (IISGS), Vigo, Spain

Acknowledgements The authors thank the researchers of the RELESSER group and Research Unit of Spanish Society of Rheumatology, who carried out the original study from which SLESIS was derived (Ref 17). BTS, CW, DI and AR work in a department supported by the National Institute for Health Research University College London Hospitals Biomedical Research Centre.

Contributors AR and BTS designed the study. IR-F, JMP-R and VdC developed the SLESIS score and advised on design of the study. BTS, AR, CW and DI collected the data. BTS and VdC carried out the statistical analysis. BTS, JMP-R, IR-F, VdC, DI and AR all took part in interpreting the results and writing the final manuscript.

Funding This work was supported by the National Institute for Health Research University College London Hospitals Biomedical Research Centre and the Spanish Foundation of Rheumatology (FER). 
Competing interests None declared.

Patient consent for publication Not required.

Provenance and peer review Not commissioned; externally peer reviewed.

Data sharing statement No additional data are available.

Open access This is an open access article distributed in accordance with the Creative Commons Attribution Non Commercial (CC BY-NC 4.0) license, which permits others to distribute, remix, adapt, build upon this work non-commercially, and license their derivative works on different terms, provided the original work is properly cited, appropriate credit is given, any changes made indicated, and the use is non-commercial. See: http://creativecommons.org/licenses/by-nc/4.0/.

\section{REFERENCES}

1. Iliopoulos AG, Tsokos GC. Immunopathogenesis and spectrum of infections in systemic lupus erythematosus. Semin Arthritis Rheum 1996;25:318-36.

2. Goldblatt F, Chambers S, Rahman A, et al. Serious infections in British patients with systemic lupus erythematosus: hospitalisations and mortality. Lupus 2009;18:682-9.

3. Cervera R, Khamashta MA, Font J, et al. Morbidity and mortality in systemic lupus erythematosus during a 5-year period. A multicenter prospective study of 1,000 patients. European Working Party on Systemic Lupus Erythematosus. Medicine 1999;78:167-75.

4. Jacobsen S, Petersen J, Ullman S, et al. A multicentre study of 513 Danish patients with systemic lupus erythematosus. II. Disease mortality and clinical factors of prognostic value. Clin Rheumatol 1998;17:478-84.

5. Hernández-Cruz B, Tapia N, Villa-Romero AR, et al. Risk factors associated with mortality in systemic lupus erythematosus. A case-control study in a tertiary care center in Mexico City. Clin Exp Rheumatol 2001;19:395-401.

6. Kang I, Park SH. Infectious complications in SLE after immunosuppressive therapies. Curr Opin Rheumatol 2003;15:528-34.

7. Molina H. Update on complement in the pathogenesis of systemic lupus erythematosus. Curr Opin Rheumatol 2002;14:492-7.

8. Garred P, Voss A, Madsen HO, et al. Association of mannosebinding lectin gene variation with disease severity and infections in a population-based cohort of systemic lupus erythematosus patients. Genes Immun 2001;2:442-50.

9. Cuchacovich R, Gedalia A. Pathophysiology and clinical spectrum of infections in systemic lupus erythematosus. Rheum Dis Clin North Am 2009;35:75-93.

10. Ucar Angulo E, Rivera García N. [Comorbidity of systemic lupus erythematosus]. Reumatol Clin 2008;4(Suppl 1):17-21.

11. Zandman-Goddard G, Shoenfeld Y. Infections and SLE. Autoimmunity 2005;38:473-85.

12. Fessler BJ. Infectious diseases in systemic lupus erythematosus: risk factors, management and prophylaxis. Best Pract Res Clin Rheumatol 2002;16:281-91.

13. Danza A, Ruiz-Irastorza G. Infection risk in systemic lupus erythematosus patients: susceptibility factors and preventive strategies. Lupus 2013;22:1286-94.

14. Falagas ME, Manta KG, Betsi GI, et al. Infection-related morbidity and mortality in patients with connective tissue diseases: a systematic review. Clin Rheumatol 2007;26:663-70.
15. Edwards CJ, Lian TY, Badsha $\mathrm{H}$, et al. Hospitalization of individuals with systemic lupus erythematosus: characteristics and predictors of outcome. Lupus 2003;12:672-6.

16. Ward MM. Avoidable hospitalizations in patients with systemic lupus erythematosus. Arthritis Rheum 2008;59:162-8.

17. Rúa-Figueroa Í, López-Longo J, Galindo-Izquierdo M, et al. Incidence, associated factors and clinical impact of severe infections in a large, multicentric cohort of patients with systemic lupus erythematosus. Semin Arthritis Rheum 2017;47:38-45.

18. Zink A, Manger B, Kaufmann J, et al. Evaluation of the RABBIT Risk Score for serious infections. Ann Rheum Dis 2014;73:1673-6.

19. Rúa-Figueroa I, López-Longo FJ, Calvo-Alén J, et al. Nationa registry of patients with systemic lupus erythematosus of the Spanish Society of Rheumatology: objectives and methodology. Reumatol Clin 2014;10:17-24.

20. Pego-Reigosa JM, Rúa-Figueroa Í, López-Longo FJ, et al. Analysis of disease activity and response to treatment in a large Spanish cohort of patients with systemic lupus erythematosus. Lupus 2015;24:720-9.

21. Katz JD, Senecal JL, Rivest C, et al. A simple severity of disease index for systemic lupus erythematosus. Lupus 1993;2:119-23.

22. Hochberg MC. Updating the American College of Rheumatology revised criteria for the classification of systemic lupus erythematosus. Arthritis Rheum 1997;40:1725.

23. Cervera R, Khamashta MA, Font J, et al. Morbidity and mortality in systemic lupus erythematosus during a 10-year period: a comparison of early and late manifestations in a cohort of 1,000 patients. Medicine 2003:82:299-308.

24. Crowson CS, Hoganson DD, Fitz-Gibbon PD, et al. Development and validation of a risk score for serious infection in patients with rheumatoid arthritis. Arthritis Rheum 2012;64:2847-55.

25. Beça S, Rodríguez-Pintó I, Alba MA, et al. Development and validation of a risk calculator to differentiate flares from infections in systemic lupus erythematosus patients with fever. Autoimmun Rev 2015;14:586-93.

26. Goldblatt F, Yuste J, Isenberg DA, et al. Impaired C3b/iC3b deposition on Streptococcus pneumoniae in serum from patients with systemic lupus erythematosus. Rheumatology 2009;48:1498-501.

27. Duffy KN, Duffy CM, Gladman DD. Infection and disease activity in systemic lupus erythematosus: a review of hospitalized patients. J Rheumatol 1991;18:1180-4.

28. Noël V, Lortholary $\mathrm{O}$, Casassus $\mathrm{P}$, et al. Risk factors and prognostic influence of infection in a single cohort of 87 adults with systemic lupus erythematosus. Ann Rheum Dis 2001;60:1141-4.

29. Ruiz-Irastorza G, Olivares N, Ruiz-Arruza I, et al. Predictors of major infections in systemic lupus erythematosus. Arthritis Res Ther 2009;11:R109.

30. Pryor BD, Bologna SG, Kahl LE. Risk factors for serious infection during treatment with cyclophosphamide and high-dose corticosteroids for systemic lupus erythematosus. Arthritis Rheum 1996;39:1475-82.

31. Gladman DD, Hussain F, Ibañez D, et al. The nature and outcome of infection in systemic lupus erythematosus. Lupus 2002;11:234-9.

32. Sisó A, Ramos-Casals M, Bové A, et al. Previous antimalarial therapy in patients diagnosed with lupus nephritis: influence on outcomes and survival. Lupus 2008;17:281-8.

33. Bultink IE, Hamann D, Seelen MA, et al. Deficiency of functional mannose-binding lectin is not associated with infections in patients with systemic lupus erythematosus. Arthritis Res Ther 2006;8:R183. 